\title{
Consequências da automedicação em portadores de insuficiência renal
}

\author{
Consequences of self-medication in renal insufficiency patients \\ Consecuencias de la automedicación en pacientes con insuficiencia renal
}

Recebido: 18/11/2021 | Revisado: 27/11/2021 | Aceito: 29/11/2021 | Publicado: 05/12/2021

\author{
Gênisson Souza Silva \\ ORCID: https://orcid.org/0000-0002-3817-7579 \\ Faculdade Independente do Nordeste, Brasil \\ E-mail: genisson755@gmail.com \\ Ivana Santos Ribeiro \\ ORCID: https://orcid.org/0000-0002-4688-7212 \\ Faculdade Independente do Nordeste, Brasil \\ E-mail: ivie.sribeiro@gmail.com \\ Matheus Santos Marques \\ ORCID: https://orcid.org/0000-0001-9734-1001 \\ Faculdade Independente do Nordeste, Brasil \\ E-mail: matheusmarques@ fainor.com.br
}

\begin{abstract}
Resumo
A automedicação refere-se ao consumo de medicamentos para tratamento de doenças e sintomas, decorrentes de uma autoavaliação, sem participação de médico ou profissional habilitado. Essa prática apesar de compreendida como parte do autocuidado, quando realizada de forma negligente representa um grande risco. Os efeitos dessa prática podem ocasionar desde interações medicamentosas e reações adversas, até casos graves de intoxicação levando à morte. Logo, o presente estudo tem por objetivos verificar os índices da automedicação em portadores de insuficiência renal, descrever suas consequências, analisar suas principais causas e a importância da atuação do farmacêutico neste contexto. Trata-se de uma pesquisa exploratória e descritiva com abordagem quali-quantitativa, realizada no período de agosto a outubro de 2021. A coleta dos dados foi realizada através da aplicação de um questionário online, fechado, estruturado, autoaplicado e anônimo, cujo modelo se insere na técnica de snowball. Posteriormente foi realizada análise e construção de tabela com parâmetros pré-definidos, discussão acerca dos resultados obtidos, bem como uma correlação com estudos disponíveis na literatura. Os resultados desvelaram que o índice da automedicação em portadores de insuficiência renal é elevado. Conclui-se é elevado o índice de pacientes renais que se automedicam, e que essa prática pode inferir negativamente no tratamento desses pacientes, ademais, a inserção do profissional farmacêutico mostrou-se primordial para redução da automedicação e melhoria da qualidade de vida, contribuindo para um tratamento mais seguro e adoção de uma terapia medicamentosa mais adequada e efetiva.
\end{abstract}

Palavras-chave: Automedicação; Farmacêutico; Insuficiência renal.

\begin{abstract}
Self-medication refers to the consumption of medication to treat diseases and symptoms, resulting from a selfassessment, without the participation of a physician or qualified professional. This practice, despite being understood as part of self-care, when negligently performed represents a great risk. The effects of this practice can cause from drug interactions and adverse reactions, to severe cases of intoxication leading to death. Therefore, this study aims to verify the rates of self-medication in patients with renal failure, describe its consequences, analyze its main causes and the importance of the pharmacist's role in this context. This is an exploratory and descriptive research with a qualiquantitative approach, carried out from august to october 2021. Data collection was carried out through the application of an online, closed, structured, self-applied and anonymous questionnaire, whose model is inserts in snowball technique. Subsequently, an analysis and construction of a table with pre-defined parameters was carried out, as well as a discussion of the results obtained, as well as a correlation with studies available in the literature. The results revealed that the rate of self-medication in patients with renal failure is high. It is concluded that the rate of kidney patients who self-medicate is high, and that this practice can negatively affect the treatment of these patients. Furthermore, the insertion of the pharmacist was essential to reduce self-medication and improve quality of life, contributing to a safer treatment and adoption of a more adequate and effective drug therapy.
\end{abstract}

Keywords: Self-medication; Pharmaceutical; Renal insufficiency.

\section{Resumen}

La automedicación se refiere al consumo de medicamentos para tratar enfermedades y síntomas, resultado de una autoevaluación, sin la participación de un médico o profesional calificado. Esta práctica, a pesar de entenderse como parte del autocuidado, cuando se realiza de forma negligente representa un gran riesgo. Los efectos de esta práctica 
pueden causar desde interacciones medicamentosas y reacciones adversas, hasta casos graves de intoxicación que conducen a la muerte. Por tanto, este estudio tiene como objetivo verificar las tasas de automedicación en pacientes con insuficiencia renal, describir sus consecuencias, analizar sus principales causas y la importancia del papel del farmacéutico en este contexto. Se trata de una investigación exploratoria y descriptiva con enfoque cuali-cuantitativo, realizada de agosto a octubre de 2021. La recolección de datos se realizó mediante la aplicación de un cuestionario en línea, cerrado, estructurado, autoaplicado y anónimo, cuyo modelo se inserta en técnica de bola de nieve. Posteriormente, se realizó un análisis y construcción de una tabla con parámetros predefinidos, así como una discusión de los resultados obtenidos, así como una correlación con los estudios disponibles en la literatura. Los resultados revelaron que la tasa de automedicación en pacientes con insuficiencia renal es alta. Se concluye que la tasa de pacientes renales que se automedican es alta, y que esta práctica puede afectar negativamente el tratamiento de estos pacientes. Además, la inserción del farmacéutico fue fundamental para reducir la automedicación y mejorar la calidad de vida, contribuyendo a un tratamiento más seguro y a la adopción de una farmacoterapia más adecuada y eficaz.

Palabras clave: Automedicación; Farmacéutico; Insuficiencia renal.

\section{Introdução}

Os rins são os órgãos responsáveis pela eliminação do excesso de líquidos e resíduos produzidos pelo corpo, controle de eletrólitos como sódio, fósforo e potássio, regulam a água, atuam na liberação de alguns hormônios responsáveis pelo controle da pressão sanguínea, na produção da série vermelha do sangue e no fortalecimento dos ossos. Além dessas funções, são encarregados de eliminar os medicamentos e toxinas ingeridos. A insuficiência renal (IR) é uma patologia sistêmica e representa a incapacidade desses órgãos de realizar essas funções, podendo ser causada por doenças como hipertensão e diabetes ou causas genéticas e hereditárias (Brasil, 2015).

O diagnóstico muitas vezes é realizado quando parte da função renal já foi comprometida, havendo uma perda massiva do funcionamento dos rins, onde ocorre a redução da taxa de filtração glomerular (TFG) (Ribeiro et al., 2008; Brasil, 2015). A IR uma vez estabelecida, é categorizada em estágios que vão do I onde há início de lesão renal, mas com TFG acima de $90 \mathrm{ml} / \mathrm{min} / 1,73 \mathrm{~m}^{2}$ ao $\mathrm{V}$ que caracteriza falência renal, onde o doente apresenta-se sintomático, necessitando de terapias substitutivas como diálise peritoneal (DP), hemodiálise (HD) ou realizar transplante, nesse estágio a TFG situa-se abaixo de $15 \mathrm{ml} / \mathrm{min} / 1,73 \mathrm{~m}^{2}$ (Egidio Romão Junior, 2004).

Os medicamentos no organismo humano são disponibilizados em quatro etapas intituladas como "ADME”: absorção, distribuição, metabolização e excreção. Em portadores de IR, a etapa de eliminação ou excreção, compreende um estágio de extrema importância, visto que a maior parte dos medicamentos são excretados por via renal. A excreção por outras vias como o suor, as fezes, o ar expirado ou ainda o leite materno, é considerada quase que desprezível quando comparadas à renal. Esse processo nos rins passa por três etapas fundamentais: filtração glomerular, secreção tubular ativa e a reabsorção passiva. Em casos de comprometimento da função renal, a posologia de diversos medicamentos necessita de adaptação ou redução. Ademais, existe o risco de toxicidade em decorrência da elevada concentração de fármacos e seus metabólitos em alguns tecidos renais (Pereira, 2007; Rang y dale: Farmacologia, 1987/2016).

Pessoas portadoras dessa patologia necessitam do uso de polifarmácia em decorrência das comorbidades associadas, como distúrbios cardiovasculares e metabólicos, tendo, portanto, uma maior probabilidade de ocorrer eventos adversos, caracterizando um problema relacionado aos medicamentos (PRM) (Marquito et al., 2014; Lemos et al., 2020). O uso de muitos medicamentos, alguns sintomas e essas comorbidades, podem levar esse grupo de pacientes a realizar automedicação devido aos riscos de desencadear outros problemas de saúde (Santana da Silva Soares et al., 2019).

Associada ao uso irracional de medicamentos, a automedicação consiste na prática de utilização de fármacos para o tratamento de doenças ou sintomas, sem a prescrição médica, diagnóstico ou acompanhamento por profissional qualificado (Duarte Vernizi \& Lange da Silva, 2016). Cerca de 35\% dos medicamentos consumidos no Brasil, são realizados através dessa prática (Aquino, 2008). 
A automedicação pode ser desenvolvida por diversos fatores como, a dificuldade no acesso aos serviços básicos de saúde, facilidade de aquisição de medicamentos devido ao grande número de farmácias, e também através do incentivo promovido por propagandas que evidenciam as vantagens da utilização dos fármacos que omitem os riscos dos mesmos. Além disso, a prática da indicação por amigos, familiares e até mesmo balconistas de farmácias, são fatores que contribuem com esse aumento (Aquino, 2008; Pereira et al., 2017).

Esse elevado índice, caracteriza um grande desafio na busca pelo uso racional de medicamentos (URM), além disso, pode desencadear diversos problemas àqueles que a realizam, por não possuírem em sua grande maioria, informações corretas e/ou necessárias para uma farmacoterapia adequada. Os riscos associados são inúmeros e incluem reação adversa ao medicamento (RAMs), intoxicação, dependência química, resistência microbiana, interações medicamentosas (IMs), interferência no tratamento de doenças já estabelecidas e até ocultação ou agravamento de doenças em curso (Faqihi \& Sayed, 2020).

Apesar de ser caracterizada como uma prática inadequada, pode ser realizada sem a prescrição médica de forma consciente, desde que esta seja feita com o auxílio de um profissional qualificado como o farmacêutico. É imprescindível que os usuários tenham conhecimento de alguns dados pertinentes ao uso dos medicamentos como a forma de utilizá-los, o tempo permitido e os efeitos adversos (World Health Organization, 1998).

Diante do exposto, esta pesquisa apresenta como objetivo, descrever as consequências da automedicação em portadores de insuficiência renal, bem como, analisar o conhecimento dos pacientes a respeito da automedicação e sobre o papel do farmacêutico nesse contexto e no desenvolvimento de estratégias de intervenção.

\section{Metodologia}

Este estudo compreendeu-se de uma pesquisa exploratória e descritiva com abordagem quali-quantitativa que buscou evidenciar consequências ocasionadas pelo uso indiscriminado de medicamentos por pessoas com IR, além de identificar o conhecimento dos mesmos acerca do papel do profissional farmacêutico nos cuidados quanto a terapia medicamentosa.

A pesquisa exploratória propicia uma maior proximidade com o problema estudado, onde é possível torná-lo mais compreensivo, e auxiliar na construção de novas conjecturas. Este tipo de estudo é mais flexível, e um de seus aspectos é a interação com pessoas que possuem algum tipo de ligação com a situação em questão. Já a pesquisa descritiva vem ser aquela, que como já sugere o nome, realiza a descrição dos aspectos relacionados ao problema pesquisado, tendo como base, a criação de uma relação entre as variáveis encontradas, sendo característico deste tipo de estudo o emprego de procedimentos padronizados de coleta de dados, como questionários (Gil, 2002).

$\mathrm{Na}$ abordagem dos dados, as análises qualitativas referem-se àquelas que vão além das variáveis, buscando-se a realização de uma discussão mais complexa, ou seja, não se pauta somente nas análises estatísticas, mas, na construção de novas hipóteses. Por outro lado, as análises quantitativas se prendem às observações rigorosas de suas variáveis, sempre com emprego de algum método matemático (Gunther, 2006).

Os participantes foram pacientes portadores de IR sendo a amostra delimitada através de critérios de inclusão e exclusão, excluídas pessoas com menos de 18 anos, diagnosticadas a menos de 1 ano e que residem fora do Brasil. A coleta dos dados se deu por meio da aplicação de um questionário "online", fechado, estruturado, autoaplicado e anônimo, tendo como método de coleta a técnica snowball, criado através do endereço eletrônico (pesquisa.farmacia.fainor@gmail.com) única e exclusivamente para a finalidade do estudo, com acesso restrito pelos autores. O questionário foi dividido em quatro blocos de questões, o primeiro pertinente às características sociodemográficas do participante, delimitando o perfil do mesmo, o segundo referente aos dados sobre a doença, o terceiro trata de questões sobre a prática da automedicação e o quarto trata sobre o conhecimento do participante acerca do papel do farmacêutico. 
A técnica snowball, compreende uma categoria de pesquisa de campo, e trata-se de uma maneira de coleta de dados de forma aleatória não probabilística, até que se chegue ao objetivo almejado, onde um participante divulga para outro e assim sucessivamente (Albuquerque, 2009).

O estudo foi dividido em duas etapas: coleta dos dados, construção e análise dos resultados. O questionário foi disponibilizado através de um link que pôde ser acessado voluntariamente pelo participante, ficando disponível entre os meses de agosto e outubro de 2021, onde o mesmo assinou o Termo de Consentimento Livre e Esclarecido (TCLE), enviado em anexo. A pesquisa foi realizada através da internet, buscando alcançar uma amostra diversificada entre diversas regiões do país e os participantes foram convidados através de mídias sociais de órgãos, instituições e associações relacionados ao tema e através de redes sociais a qual fazem parte. $\mathrm{O}$ link foi divulgado nas publicações indexadas às mídias sociais descritas, através de texto convite.

Após a coleta dos dados, foi realizada a tabulação e análise dos resultados, seguida por correlação com estudos preliminares da literatura. Para análise dos resultados, foi utilizado o "software" de processamento estatístico SPSS. Para seleção dos estudos, foram buscadas publicações em revistas com indexação na Qualis e na base de dados da divisão de ciências da Biblioteca Virtual em Saúde (BVS) - Literatura latino-americana e do Caribe em Ciências da Saúde (LILACS), PubMED e Science, além de documentos oficiais.

Antes de iniciar a coleta de dados, o projeto de pesquisa foi submetido à aprovação do Comitê de Ética em Pesquisa da Faculdade Independente do Nordeste - FAINOR, sob o parecer $n^{\circ} 4.946 .736$, respeitando os preceitos éticos da pesquisa envolvendo seres humanos.

\section{Resultados e Discussão}

\section{Caracterização sociodemográfica}

Participaram do estudo, 83 pacientes renais, com idades entre 18 e 67 anos, com média de idade de 42 anos, residentes em todo o território nacional, sendo a amostra composta por 62 pessoas do sexo feminino e 21 do sexo masculino. A região com maior número foi nordeste (40), seguida por sudeste (29), sul (7), centro-oeste (6) e apenas 1 participante da região Norte.

\section{Análise do perfil da doença}

Considerando o perfil da doença dos participantes, conforme descrito no Quadro 1, 50,6\% possuem diagnóstico a mais de 10 anos, sendo a hemodiálise $(55,4 \%)$ a forma de tratamento mais utilizada, seguido por transplante renal $(36,1 \%)$, tratamento conservador $(6,0 \%)$ e diálise peritoneal $(2,5 \%)$. 
Quadro 1. Perfil da doença dos participantes.

\begin{tabular}{|l|l|l|}
\hline \multicolumn{2}{|c|}{ Tempo de diagnóstico da Insuficiência Renal } \\
\hline & Frequência & Percentual \\
\hline Menos de 5 anos & 21 & $25,3 \%$ \\
\hline Entre 5 e 10 anos & 20 & $24,1 \%$ \\
\hline Mais de 10 anos & 42 & $50,6 \%$ \\
\hline Total & $\mathbf{8 3}$ & $\mathbf{1 0 0 , 0 \%}$ \\
\hline & \multicolumn{2}{|c|}{ Tipo de tratamento que realiza } \\
\hline & Frequência & Percentual \\
\hline Conservador & 5 & $6,0 \%$ \\
\hline Diálise Peritoneal & 2 & $2,4 \%$ \\
\hline Hemodiálise & 46 & $55,4 \%$ \\
\hline Transplante Renal & 30 & $36,1 \%$ \\
\hline Total & $\mathbf{8 3}$ & $\mathbf{1 0 0 , 0 \%}$ \\
\hline
\end{tabular}

Fonte: Dados da pesquisa (2021).

Os dados descritos coincidem com o quantitativo apontado no estudo de Neves et al. (2020), que realiza uma análise do cenário de diálise no Brasil entre os anos de 2009 - 2018, mostrando que a HD é a terapia substitutiva de função renal mais realizada (92\% em 2018), enquanto a DP obteve queda de $10,5 \%$ para 7,8\% no período do referido estudo.

Em análise realizada por Ribeiro et al. (2008) numa unidade de nefrologia, a maior parte dos pacientes (86,6\%) também realizavam tratamento por HD. Diversos estudos buscam compreender os fatores relacionados à escolha da terapia substitutiva de função renal e, em geral, os critérios adotados referem-se às características clínicas individuais dos pacientes. Em sua grande maioria, a escolha do tratamento é realizada pelo profissional médico, e as condições clínicas tendem a HD, ficando a DP indicada em situações específicas, como em casos de portadores de problemas cardíacos, diabéticos e fatores sociais. Quando as condições clínicas não influenciam, a modalidade de tratamento pode ser optativa do paciente (Santos et al., 2018; Jassal et al., 2002).

Ao analisar as causas da IR, como descreve a Quadro 2, as principais doenças de base relatadas pelos participantes foram Glomerulonefrites (18,1\%), seguida por Hipertensão Arterial (16,9\%), causas desconhecidas (12\%) e diabetes (4,8\%).

Quadro 2. Causas da Insuficiências Renal.

\begin{tabular}{|l|l|l|}
\hline Causas & Frequência & Percentual \\
\hline Glomerulonefrites & 15 & $18,1 \%$ \\
\hline Hipertensão Arterial & 14 & $16,9 \%$ \\
\hline Causa desconhecida & 10 & $12,0 \%$ \\
\hline Diabetes & 4 & $4,8 \%$ \\
\hline ITU & 4 & $4,8 \%$ \\
\hline
\end{tabular}




\begin{tabular}{|l|l|l|}
\hline Lúpus Eritematoso & 3 & $3,6 \%$ \\
\hline Nefropatia por IgA & 3 & $3,6 \%$ \\
\hline Nefrocalcinose & 3 & $3,6 \%$ \\
\hline Síndrome de Alport & 2 & $2,4 \%$ \\
\hline COVID-19 & 1 & $1,2 \%$ \\
\hline SHU & 1 & $1,2 \%$ \\
\hline Uso Abusivo de Anti Inflamatório & 1 & $1,2 \%$ \\
\hline Anemia Falciforme & 1 & $1,2 \%$ \\
\hline Rim Policístico & 1 & $1,2 \%$ \\
\hline Outras causas & 20 & $24,1 \%$ \\
\hline Total & $\mathbf{8 3}$ & $\mathbf{1 0 0 , 0 \%}$ \\
\hline
\end{tabular}

Fonte: Dados da Pesquisa (2021).

A IR não tem uma causa definida, diversos os fatores desencadeantes da patologia, que vão desde doenças primárias, até doenças de causas sistêmicas. Os dados apontados se assemelham aos dispostos em estudos, mostrando a hipertensão arterial, diabetes e glomerulonefrites como causas determinantes. A correlação entre as causas e o nível de lesão renal provocada é tema de diversas análises. A diabetes e hipertensão arterial, por exemplo, podem levar à vasoconstrição renal, danos microvasculares, isquemias, e problemas na excreção de metabólitos, que evoluem com perda progressiva da função renal. Outros fatores como raça, condições socioeconômicas, ambientais, e psicológicas podem estar relacionados ao processo de desenvolvimento, evolução e desfecho da doença (Aguiar et al., 2020; Cherchiglia et al., 2010; Rudnicki et al., 2014).

\section{Terapia medicamentosa e prática da automedicação}

Ao observar a terapia medicamentosa dos participantes, ou seja, o uso dos fármacos prescritos, relacionados no Quadro 3 , considerando aqueles pacientes que relataram utilizar entre 3 e 5 ou mais de 5 medicamentos, tem-se uma amostra de até 73,5\% de pacientes que realizam polifarmácia. Esses achados aproximam-se ao do estudo Michel et al., (2021) que ao analisar o perfil de pacientes com IR observou o percentual de 92,2\% que realizam polifarmácia.

Segundo Secoli (2010), define-se polifarmácia como a utilização de cinco ou mais medicamentos. Receituários com vários medicamentos e comorbidades associadas, leva ao uso de muitos fármacos, o que pode aumentar o risco de prescrição de fármacos desnecessários para a terapia, além do aumento dos riscos de interações, reações adversas, intoxicações medicamentosas, doenças iatrogênicas, internações hospitalares e até óbito (Pereira, et al. 2017). 
Quadro 3. Terapia medicamentosa e prática de automedicação.

\begin{tabular}{|c|c|c|c|c|c|}
\hline & & & Realiza & ledicação & \\
\hline & & & $\operatorname{sim}$ & não & \\
\hline & & $\mathrm{n}$ & 1 & 0 & 1 \\
\hline & & $\%$ do Total & $1,2 \%$ & $0,0 \%$ & $1,2 \%$ \\
\hline & & $\mathrm{n}$ & 18 & 3 & 21 \\
\hline $\begin{array}{l}\text { Quantidade de } \\
\text { Medicamentos que }\end{array}$ & & $\%$ do Total & $21,7 \%$ & $3,6 \%$ & $25,3 \%$ \\
\hline $\begin{array}{l}\text { são que utiliza } \\
\text { (prescritos) }\end{array}$ & & $\mathrm{n}$ & 17 & 10 & 27 \\
\hline & & $\%$ do Total & $20,5 \%$ & $12,0 \%$ & $32,5 \%$ \\
\hline & & $\mathrm{n}$ & 24 & 10 & 34 \\
\hline & 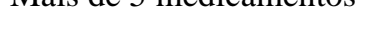 & $\%$ do Total & $28,9 \%$ & $12,0 \%$ & $41,0 \%$ \\
\hline & & $\mathrm{n}$ & 60 & 23 & 83 \\
\hline Fotal ta t t & & $\%$ do Total & $72,3 \%$ & $27,7 \%$ & $100 \%$ \\
\hline
\end{tabular}

Fonte: Dados da Pesquisa (2021).

Conforme descrito, nota-se um elevado percentual de participantes que realizam a prática da automedicação ( $\mathrm{n}=60 /$ $72,3 \%$ ), destes, $n=41 / 49,4 \%$ informam comunicar ao médico a realização da prática. Tratando-se de uma amostra composta por portadores de IR, esse dado, associado ao índice de participantes polifarmácia compõe um quadro preocupante. A automedicação realizada de forma negligente, já representa uma prática com potenciais riscos à saúde e a realização desta prática por portadores de comorbidades aumenta a possibilidade da ocorrência desses efeitos, até mesmo com consequências mais graves.

Portadores de IR em sua maioria utilizam polifarmácia, apresentam debilidades decorrentes da necessidade da diálise, além da possibilidade de um conjunto de doenças como anemia, hipoparatireoidismo, inflamação crônica, diabetes, hipertensão arterial, desmineralização óssea e dislipidemias. Esses fatores, associados à queda ou déficit da função renal, tornam estes pacientes vulneráveis aos efeitos negativos da automedicação, como superdosagem e intoxicação medicamentosa (Lemos et al., 2020).

A prescrição de múltiplos medicamentos é comum na rotina clínica, principalmente em se tratando de pacientes portadores de doenças crônicas. Essas prescrições muitas das vezes ocasionam interações medicamentosas, que algumas vezes já são previstas e podem até trazer benefícios, como aumento do efeito ou absorção do fármaco concomitante, em outras, porém, essas interações ocasionam efeitos que podem interferir negativamente em terapias adjacentes, no desenvolvimento de doenças, vícios e problemas relacionados, tornando-a um dos problemas relacionados ao medicamento (PRM) mais comuns e com maiores impactos na vida do paciente (Marquito et al., 2014).

Os principais motivos que levam ao uso da automedicação relatados pelos participantes da pesquisa foram: somente dor $(n=31 / 37,3 \%)$, dor, problemas gástricos e febre $(n=1 / 26,5 \%)$, seguidos por somente febre $(n=2 / 2,4 \%)$, somente problemas gástricos ( $n=1 / 1,2 \%)$, outros $(n=4 / 4,8 \%)$ e não se automedicam $(n=23 / 27,7 \%)$.

Compreender os motivos que levam a automedicação faz-se necessário para o desenvolvimento de ações que visam minimizar os danos referentes a essa prática. É importante entender que a responsabilidade não deve ser imputada somente aos consumidores, o incentivo da mídia e autoridades públicas, a facilidade de aquisição, a sobrecarga dos serviços de saúde e a falta 
de conhecimento e orientações adequadas se somam ao conjunto de causas que favorecem o crescimento dos indicadores da automedicação (Constantino et al., 2020; Melo et al., 2021).

As classes dos medicamentos que os participantes do estudo relataram fazer uso por conta própria foram analgésicos $(n=23 / 27,7 \%)$, antitérmicos $(n=2 / 2,4 \%)$, anti-inflamatórios $(=2 / 2,4 \%)$, antialérgicos $(n=2 / 2,4 \%)$, antiácidos $(n=1 / 1,2 \%)$ e antibióticos $(n=1 / 1,2 \%)$. Alguns ainda relataram que fazem uso concomitante de mais de uma classe desses medicamentos, representando uma amostra de ( $n=29 / 34,9 \%)$, o que eleva os riscos de IM e o potencial efeito nefrotóxico, agravando o quadro da insuficiência renal e/ou desencadeando outros problemas.

Em análise, o perfil farmacoterapêutico de pacientes com IR e hemodialíticos em uma clínica da Bahia, Lemos et al. (2020) aponta que cerca de $40 \%$ dos participantes relataram realizar automedicação, sendo os medicamentos mais utilizados aqueles que atuam no sistema digestivo e metabolismo, seguidos por medicamentos para o sistema cardiovascular e para o musculoesquelético.

Muitas vezes, o uso de medicamentos por conta própria advém da busca por diminuir os efeitos debilitantes das terapias implementadas, ou em sanar os efeitos adversos de medicamentos prescritos, o que pode ocorrer pela não orientação adequada aos pacientes, e pela necessidade de acompanhamento farmacêutico, o profissional qualificado para análise da terapia medicamentosa de cada paciente.

\section{Percepção dos participantes sobre a atuação do profissional Farmacêutico}

No que se refere a percepção dos participantes sobre a atuação do profissional farmacêutico, obtiveram-se os seguintes resultados conforme descreve o Quadro 4, 77,1\% relataram ter conhecimento do papel do farmacêutico como profissional habilitado a fornecer orientações a respeito de medicamentos, bem como ser o responsável pela dispensação correta. No entanto, $51,8 \%$ alegaram não ter costume de pedir orientação, e 72,3\% nunca realizaram consulta ou acompanhamento com este profissional.

Esses dados demonstram a necessidade do reconhecimento deste profissional para além de dispensador de medicamentos, com um papel de grande relevância na atuação em assistência à saúde. Maia et al. (2018) mostram em seu estudo a importância da atuação farmacêutica em clínicas de hemodiálise, em especial na orientação farmacoterapêutica para administração de medicamentos, que em cerca de $26,3 \%$ ocorre de forma errada. O estudo aponta ainda que a atuação do farmacêutico pode trazer diversos benefícios como redução das IMs e PRM, melhoria de custos relacionados a medicamentos e correta orientação seja a profissionais ou pacientes.

Quadro 4. Percepção dos participantes a respeito do farmacêutico.

\begin{tabular}{|l|l|l|l|l|l|l|l|}
\hline \multirow{3}{*}{} & & \multicolumn{3}{|l|}{$\begin{array}{l}\text { Conhecimento sobre o } \\
\text { papel do farmacêutico }\end{array}$} & \multicolumn{2}{l|}{$\begin{array}{l}\text { Pede orientação ao } \\
\text { farmacêutico }\end{array}$} & \multicolumn{2}{l|}{$\begin{array}{l}\text { Realizou consulta } \\
\text { /acompanhamento com } \\
\text { Farmacêutico }\end{array}$} \\
\cline { 2 - 9 } & & Sim & Não & Sim & Não & Sim & Não \\
\cline { 2 - 8 } & $\mathrm{n}$ & 64 & 19 & 40 & 43 & 23 & 60 \\
\cline { 2 - 9 } & $\%$ & $\mathbf{7 7 , 1 \%}$ & $22,9 \%$ & $48,2 \%$ & $\mathbf{5 1 , 8 \%}$ & $27,7 \%$ & $\mathbf{7 2 , 3 \%}$ \\
\hline Total & $\mathrm{n} / \%$ & $83 / 100 \%$ & $83 / 100 \%$ & $83 / 100 \%$ & \\
\hline
\end{tabular}

Fonte: Dados da Pesquisa (2021). 
Em se tratando da contribuição farmacêutica na redução de PRM e na adesão terapêutica, Sánchez-Gili et al. (2011) mostra em seu estudo, que ao analisar 94 pacientes com IR observou-se uma abundante quantidade de PRMs, 52 encontrados, que representa um grande risco à eficácia terapêutica e segurança do paciente, ademais, mostra que a intervenção farmacêutica nestes pacientes conseguiu aumentar de forma estatisticamente significativa a adesão medicamentosa passando de 78,6\% para $87,8 \%$.

Ao relacionar o índice de pacientes que realizam automedicação com os que pedem orientação ao farmacêutico, conforme descreve o Quadro 5, é notável que dentre os pacientes que realizam essa prática, a maioria ( $\mathrm{n}=33 / 55 \%)$ não pedem orientação ao farmacêutico sobre os medicamentos, em contrapartida, entre os que não se automedicam, a maior parte (n=13/ 56,5\%) pedem orientação. Estes dados demonstram que o nível de conhecimento adquirido pelos participantes, bem como a atuação do farmacêutico quanto a orientação adequada impactam diretamente os índices de automedicação.

Quadro 5. Correlação dos índices de automedicação e orientação farmacêutica.

\begin{tabular}{|c|c|c|c|c|c|}
\hline & & & Pede o & ao farm: & bre medicamentos \\
\hline & & & Sim & Não & Total \\
\hline & & $\mathrm{n}$ & 27 & 33 & \\
\hline & & $\%$ & $45,0 \%$ & $55,0 \%$ & \\
\hline & & $\mathrm{n}$ & 13 & 10 & \\
\hline & nav & $\%$ & $56,5 \%$ & $43,5 \%$ & \\
\hline & & $\mathrm{n}$ & 40 & 43 & 83 \\
\hline & & $\%$ & $48,2 \%$ & $51,8 \%$ & $100 \%$ \\
\hline
\end{tabular}

Fonte: Dados da Pesquisa (2021).

Nesse sentido, Simões Fernandes e César Cembranelli (2015) demonstraram em seu estudo a importância do farmacêutico quanto à orientação ao paciente, dado que este possui os conhecimentos em saúde e medicamentos, podendo fornecer informações seguras aos pacientes, respeitando a terapia implementada, bem como suas individualidades. Além disso, o autor reitera a importância da legalização da prescrição farmacêutica, e como esta é uma valiosa ferramenta na redução dos índices de automedicação, sendo o farmacêutico um profissional de acesso mais viável e rápido.

A atuação do profissional farmacêutico dentro de uma equipe multidisciplinar nesse contexto do acompanhamento a portadores de IRC, se mostra de extrema importância, visto que auxiliando na terapia medicamentosa e em todos os níveis de atenção, pode ajudar a retardar a progressão da doença e evitar potenciais riscos ocasionados pelo uso de polifarmácia, além de minimizar as hipóteses de possíveis PRMs, bem como ajudar os pacientes na adesão ao tratamento (Campos et al., 2021). Conforme descrito na Resolução n. ${ }^{\circ} 500$ (2009), o farmacêutico pode atuar nos serviços de diálise, com a equipe, lidando diretamente com os pacientes, realizando acompanhamento da farmacoterapia prescrita, contribuindo para um serviço de qualidade, desenvolvendo funções administrativas e podendo incentivar a promoção do URM, com medidas e campanhas socioeducativas para pacientes e cuidadores. 


\section{Considerações Finais}

Após análise dos resultados é possível inferir que o índice da automedicação em portadores de insuficiência renal é elevado, apesar das sérias consequências que esta prática pode ocasionar como o aumento do risco de IMs e RAMs, intoxicações, desenvolvimento de doenças iatrogênicas, aumento de hospitalizações, diminuição da efetividade do tratamento, agravamento da lesão renal e em casos graves pode levar a morte, portanto, os objetivos deste estudo alcançaram os resultados almejados, reafirmando a necessidade de acompanhamento com profissional farmacêutico para este grupo de pacientes. Foram evidenciadas como causas da automedicação: os efeitos adversos decorrentes dos tratamentos realizados, comorbidades e complicações ocasionadas pela insuficiência renal, a falta de conhecimento por parte dos pacientes sobre os prejuízos dessa prática para os rins e a ausência de um acompanhamento farmacoterapêutico.

Nesse contexto, é desvelada a importância da atuação do farmacêutico, de modo a contribuir para redução do índice da automedicação e os problemas associados, auxiliando na melhoria da qualidade de vida dos pacientes portadores de IR, bem como, contribuindo para um tratamento mais efetivo, e adoção de uma terapia medicamentosa mais segura e adequada. Esperase, com os resultados desta pesquisa, demonstrar a importância da inserção do profissional farmacêutico nas equipes multidisciplinares de saúde no acompanhamento a pacientes renais em todos os estágios de tratamento e em todos os níveis de atenção, visto que o farmacêutico é o profissional capacitado a identificar PRMs e IMs, auxiliar no manejo e resolução dos mesmos, bem como prestar às orientações adequadas para a promoção do URM. Vale ratificar que este estudo se mostra de grande relevância, apresentando resultados promissores para posteriores trabalhos que buscam estratégias de controle e acompanhamento farmacoterapêutico, com o intuito de otimizar a terapia medicamentosa para pacientes renais. Ademais, faz-se necessário mais estudos que busquem avaliar os efeitos do uso indiscriminado de medicamentos no agravamento da lesão renal, que elucidem o metabolismo e excreção dos mesmos nesse grupo de pacientes e as principais classes de fármacos que podem deteriorar a função renal.

\section{Agradecimentos}

A todos que direta ou indiretamente contribuíram para a realização e sucesso do artigo.

\section{Referências}

Aguiar, L. K. d., Prado, R. R., Gazzinelli, A., \& Malta, D. C. (2020). Fatores associados à doença renal crônica: inquérito epidemiológico da Pesquisa Nacional de Saúde. Revista Brasileira de Epidemiologia, 23. https://doi.org/10.1590/1980-549720200044.

Albuquerque, E. M. d. (2009). Avaliação da técnica de amostragem respondent-driven sampling na estimação de prevalências de doenças transmissíveis em populações organizadas em redes complexas [PublishedVersion, reponame:Repositório Institucional da FIOCRUZ]. https://www.arca.fiocruz.br/handle/icict/2411

Aquino, D. S. d. (2008). Por que o uso racional de medicamentos deve ser uma prioridade? Ciência \& Saúde Coletiva, 13(suppl), 733-736. https://doi.org/10.1590/s1413-81232008000700023.

Brasil. (2015, 13 de maio). Você sabe o que é insuficiência renal? Ministério da Saúde / Blog da Saúde. http://www.blog.saude.gov.br/zfa9rd.

Campos, F., Raquel Uhdich Kleibert, K., Renz Pretto, C., Barros Klein, K., Miladi Fernandes Stumm, E., \& de Fátima Colet, C. (2021). Use of drugs and potential drug interactions in chronic renal patients in hemodialysis. Revista Saúde (Santa Maria), 47(1), Artigo e40770. https://doi.org/10.5902/223658340770.

Carvalho Michel, N. C. M., Schwartz, E., Pozza dos Santos, B., \& Lise, F. (2021). O uso dos fármacos na doença renal crônica pelos pacientes em hemodiálise. Revista Saúde em Redes, 7(1), 1-11. https://doi.org/10.18310/2446-48132021v7n1.3012g632.

Cherchiglia, M. L., Machado, E. L., Szuster, D. A. C., Andrade, E. I. G., Acúrcio, F. d. A., Caiaffa, W. T., Sesso, R., Guerra Junior, A. A., Queiroz, O. V. d., \& Gomes, I. C. (2010). Perfil epidemiológico dos pacientes em terapia renal substitutiva no Brasil, 2000-2004. Revista de Saúde Pública, 44(4), 639-649. https://doi.org/10.1590/s0034-89102010000400007. Acesso em: 4/11/2021.

Constantino, V. M., Fregonesi, B. M., Tonani, K. A. d. A., Zagui, G. S., Toninato, A. P. C., Nonose, E. R. d. S., Fabriz, L. A., \& Segura-Muñoz, S. I. (2020). Estoque e descarte de medicamentos no domicílio: Uma revisão sistemática. Ciência \& Saúde Coletiva, 25(2), 585-594. https://doi.org/10.1590/141381232020252.10882018 . 
Dispõe sobre as atribuições do farmacêutico no âmbito dos Serviços de Diálise, de natureza pública ou privada, Resolução $\mathrm{n}^{\circ}$ 500 (2009) (Brasil). https://www.cff.org.br/userfiles/file/resolucoes/500.pdf

Duarte Vernizi, M., \& Lange da Silva, L. (2016). A prática de automedicação em adultos e idosos: Uma revisão de literatura. Revista Saúde e Desenvolvimento, 10(5). https://www.revistasuninter.com/revistasaude/index.php/saudeDesenvolvimento/article/view/579.

Egidio Romão Junior, J. (2004). Doença renal crônica: Definição, epidemiologia e classificação. Jornal Brasileiro de Nefrologia, $26(3)$, 1-3. https://bjnephrology.org/wp-content/uploads/2019/11/jbn_v26n3s1a02.pdf

Eler Maia, D. E. M., Moreira Alves, S., \& Cardoso, G. (2018). Revisão bibliográfica: Atenção farmacêutica nos pacientes em hemodiálise. Brazilian Journal of Surgery and Clinical Research, 21(2) 135-139. https://www.mastereditora.com.br/periodico/20180103_170647.pdf Acesso em: 7/11/021.

Faqihi, A. H. M. A., \& Sayed, S. F. (2020). Self-medication practice with analgesics (NSAIDs and acetaminophen), and antibiotics among nursing undergraduates in University College Farasan Campus, Jazan University, KSA. Annales Pharmaceutiques Françaises. https://doi.org/10.1016/j.pharma.2020.10.012.

Gil, A. C. (2002). - Como elaborar projetos de pesquisa (6.). Atlas. (Original work published 1946).

Günther, H. (2006). Pesquisa qualitativa versus pesquisa quantitativa: esta é a questão? Psicologia Teoria e Pesquisa, 22(2), 201-209. https://doi.org/10.1590/s0102-37722006000200010.

Jassal, S. V., Krishna, G., Mallick, N. P., \& Mendelssohn, D. C. (2002). Attitudes of British Isles nephrologists towards dialysis modality selection: A questionnaire study. Nephrology Dialysis Transplantation, 17(3), 474-477. https://doi.org/10.1093/ndt/17.3.474.

Lemos, L. B., Moraes, G. S., Lemos, G. d. S., \& Nery, A. A. (2020). Automedicação em pacientes renais crônicos hemodialíticos. Revista Brasileira em Promoção da Saúde, 33, 1-10. https://doi.org/10.5020/18061230.2020.9906.

Marquito, A. B., Fernandes, N. M. d. S., Colugnati, F. A. B., \& Paula, R. B. d. (2014). Identifying potential drug interactions in chronic kidney disease patients Jornal Brasileiro de Nefrologia, 36(1), 26-34. https://doi.org/10.5935/0101-2800.20140006.

Melo, J. R. R., Duarte, E. C., Moraes, M. V. d., Fleck, K., \& Arrais, P. S. D. (2021). Automedicação e uso indiscriminado de medicamentos durante a pandemia da COVID-19. Cadernos de Saúde Pública, 37(4). https://doi.org/10.1590/0102-311x00053221.

Neves, P. D. M. d. M., Sesso, R. d. C. C., Thomé, F. S., Lugon, J. R., \& Nasicmento, M. M. (2020). Brazilian Dialysis Census: Analysis of data from the 20092018 decade. Brazilian Journal of Nephrology, 42(2), 191-200. https://doi.org/10.1590/2175-8239-jbn-2019-0234.

Pereira, D. G. (2007). Importância do metabolismo no planejamento de fármacos. Química Nova, 30(1), 171-177. https://doi.org/10.1590/s010040422007000100029

Pereira, F. G. F., Araújo, M. D. J. P., Pereira, C. R., Nascimento, D. D. S., Galiza, F. T. d., \& Benício, C. D. A. V. (2017). Automedicação em idosos ativos. Revista De Enfermagem UFPE on Line, 11(12), 4919. https://doi.org/10.5205/1981-8963-v11i12a22289p4919-4928-2017.

Rang y dale: Farmacologia (Gea Consultoría Editorial, Trad.; 8ª ed.). (2016). Elsevier. (Obra original publicada em 1987).

Ribeiro, R. d. C. H. M., Oliveira, G. A. S. A. d., Ribeiro, D. F., Bertolin, D. C., Cesarino, C. B., Lima, L. C. E. Q. d., \& Oliveira, S. M. d. (2008). Caracterização e etiologia da insuficiência renal crônica em unidade de nefrologia do interior do Estado de São Paulo. Acta Paulista de Enfermagem, 21 (spe), $207-211$. https://doi.org/10.1590/s0103-21002008000500013

Rudnicki, T. (2014). Doença renal crônica: Vivência do paciente em tratamento de hemodiálise. Contextos Clínicos, 7(1). https://doi.org/10.4013/ctc.2014.71.10.

Sánchez-Gili, M., Toro-Chico, P., Pérez-Encinas, M., Gómez-Pedrero, A. M., \& Portolés-Pérez, J. M. (2011). Intervención farmacéutica en la adherencia al tratamiento de pacientes con enfermedad renal crónica. Revista de Calidad Asistencial, 26(3), 146-151. https://doi.org/10.1016/j.cali.2010.11.012.

Santana da Silva Soares, L., Soares de Brito, E., Vitória da Silva, E., \& Galato, D. (2019). Eventos adversos relacionados ao uso de imunossupressores em pacientes transplantados. Boletim Farmacoterapeutica, (3), 11-17. http://crfmt.org.br/wp-content/uploads/2019/11/2628-8937-1-SM.pdf.

Santos, V. F. C. d., Borges, Z. N., Lima, S. O., \& Reis, F. P. (2018). Percepções, significados e adaptações à hemodiálise como um espaço liminar: A perspectiva do paciente. Interface - Comunicação, Saúde, Educação, 22(66), 853-863. https://doi.org/10.1590/1807-57622017.0148.

Secoli, S. R. (2010). Polifarmácia: Interações e reações adversas no uso de medicamentos por idosos. Revista Brasileira de Enfermagem, 63(1), 136-140. https://doi.org/10.1590/s0034-71672010000100023

Simões Fernandes, W., \& César Cembranelli, J. C. C. (2015). Automedicação e o uso irracional de medicamentos: O papel do profissional farmacêutico no combate a essas práticas. Revista Univap, 21(37), 5-11. http://revista.univap.br/index.php/revistaunivap/article/view/265/259

World Health Organization. (1998). The role of the pharmacist in self-care and self-medication (Report). WHO. 